\title{
GCU
}

Glasgow Caledonian

University

University for the Common Good

\section{Optical wireless communication based two receiver indoor positioning algorithm}

Ajmani, Manisha; Boutaleb, Tuleen; Sinanovic, Sinan

Published in:

13th International Wireless Communications and Mobile Computing Conference (IWCMC 2017)

DOI:

10.1109/IWCMC.2017.7986362

Publication date:

2017

Document Version

Author accepted manuscript

Link to publication in ResearchOnline

Citation for published version (Harvard):

Ajmani, M, Boutaleb, T \& Sinanovic, S 2017, Optical wireless communication based two receiver indoor positioning algorithm. in 13th International Wireless Communications and Mobile Computing Conference (IWCMC 2017)., 7986362, IEEE, pp. 653-658, 13th International Wireless Communications and Mobile Computing Conference (IWCMC), Valencia, Spain, 26/06/17. https://doi.org/10.1109/IWCMC.2017.7986362

\section{General rights}

Copyright and moral rights for the publications made accessible in the public portal are retained by the authors and/or other copyright owners and it is a condition of accessing publications that users recognise and abide by the legal requirements associated with these rights.

Take down policy

If you believe that this document breaches copyright please view our takedown policy at https://edshare.gcu.ac.uk/id/eprint/5179 for details of how to contact us. 


\title{
Optical Wireless Communication based Two Receiver Indoor Positioning Algorithm
}

\author{
Manisha Ajmani*, Tuleen Boutaleb* and Sinan Sinanović* \\ *School of Engineering and Built Environment, Glasgow Caledonian University, Glasgow, G4 0BA,UK \\ Email: (manisha.ajmani, t.boutaleb, sinan.sinanovic)@gcu.ac.uk
}

\begin{abstract}
This research focuses on developing a positioning algorithm for Optical Wireless Communication (OWC) based indoor positioning systems. The proposed Two Receiver Indoor Positioning (TRIP) algorithm has the potential to overcome the light beam overlapping problem and locate the position of an object in an indoor environment precisely. The current research builds upon a previously proposed positioning algorithm named Optimal Beam Radius Indoor Positioning (OBRIP) algorithm and proposes the use of two receivers to detect the optical signal to further reduce the error in position estimation while also investigating the best values for important parameters like beam radius of transmitting Light-Emitting Diodes (LED), grid size of LED array and number of LEDs in an array for multiple room shapes. The performance analysis of TRIP algorithm and subsequent comparison with the performance of OBRIP algorithm is also carried out in this research using MATLAB simulations. From the simulation results, this algorithm proves to be effective for a room with floor dimensions of $10 \mathrm{~m} \times 10$ $m$ yielding an average error in position estimation whose value is $0.69 \mathrm{~m}$ and surpasses the performance of the OBRIP with $30 \%$ lesser average error values. Using this technology for indoor positioning and tracking in the medical sector, and extending help to elderly dementia affected patients is a noble and intriguing idea.
\end{abstract}

Keywords-Indoor positioning system; Optical wireless communication; Visible light communication; Light-emitting diode; Position estimation; Beam radius; Performance analysis;

\section{INTRODUCTION}

Indoor positioning and tracking systems have long been considered as the 'revolutionary product' which will change the way of operation of indoor establishments like large convenience stores, manufacturing facilities and the medical sector. These systems are especially useful in the indoor environments like hospitals where the Indoor Positioning Systems (IPS) can be used to quickly locate health care staff, large medical equipments or even track the physical movement of patients to help ensure their safety. Much progress has been made which has led to the design and development of several indoor positioning systems, but owing to factors like high cost of implementation, low accuracy, and several assumptions while designing the system which might not hold in the practical implementation of the system [1], there is still a lot of ground left to cover.

Positioning has been classified into two categories based on the environment of operation, namely Outdoor positioning and Indoor positioning [2]. Global Positioning System (GPS), a satellite based positioning system offering a large coverage area, is regarded as the best known technology for the outdoor environment [3], [4], [5], [6], but considering that it suffers from multi-path interference and attenuation [5], [7], [8] it has less potential for being used in the indoor environment for the purpose of tracking and positioning [9], [10]. Other technologies which are based on radio frequency and ultrasound have to combat with effects like interference and noise reducing the accuracy of the system [10], [11], or require additional infrastructure which increases the overall cost of implementation of the positioning system [5], [12], [13]. A new technology, Visible Light Communication (VLC), which uses LEDs has emerged as the potential solution for the indoor positioning systems. This technology is based on the principle of optical wireless communication in which the data to be transmitted modulates the light emitted by the LED [14]. The LED emission used for this technology can be in the visible light as well as the infrared spectrum. If an IPS is designed using VLC it can be used for illumination and data transmission simultaneously [15] omitting the requirement for additional infrastructure, thus proving to be a low-cost alternative . Other major advantages of of using VLC for IPS include high security, absence of radio interference, high accuracy and large bandwidth capacity [16], [17].

Though this system paves the way for exciting possibilities in the indoor positioning sector, but for it to provide the expected results some challenges in the practical implementation of this system have to be overcome. One of the major challenges include designing a positioning algorithm which can be used alongside this Optical Wireless Communication (OWC)-based IPS. Owing to the problem of interfering or overlapping light beams from the LEDs in the transmitting LED array of the system the individual signals cannot be separated at the optical receiver which lead to loss of information or erroneous data. The Multiple LED Estimation Model (MLEM) [18] tries to solve this problem of overlapping light beams to a large extent by encoding positional information using Pulse Duration Multiplexing (PDM) and modulating the data to a higher frequency using On-Off keying (OOK) modulation. Considering that interference in the overlapping light regions can be warded off using the MLEM model, a new algorithm, Optimal Beam Radius Indoor Positioning (OBRIP) algorithm, has been proposed in related research [19]. The algorithm translates the problem of overlapping light beam regions and uses it to improve the accuracy in position estimation for 
a device inside the room with pre-installed LEDs at fixed positions. From the OBRIP algorithm the average error in position estimation is reduced to the order of a small fractional component of the size of the room, but there is still a margin for further reduction of error and increase in accuracy of the overall system. To improve these statistics, in this research the TRIP algorithm, an improvement over the OBRIP algorithm, is proposed and the use of two optical receivers is suggested for position estimation.

The organization of the remaining paper is as follows: In Section II the concept of the Two Receiver Indoor Positioning (TRIP) algorithm is introduced and its operation is discussed in detail. In Section III the simulation setup used for the performance analysis of TRIP algorithm along with the results obtained from simulation in MATLAB are discussed. Side-byside comparison with the performance of OBRIP algorithm has also been done in each sub-section. Finally, in Section IV conclusion of the research is presented.

\section{Two Receiver Indoor Positioning Algorithm} (TRIP)

The TRIP algorithm can be used in a room with a OWC-based indoor positioning system having an array of pre-positioned LEDs installed. The LEDs in the array periodically transmit an optical signal containing their location coordinates. The person (or receiver object) whose position is to be determined has two optical receivers installed on each of their shoulders (or upper corners). When this person enters the room with the IPS system, each of the receivers placed on the shoulders receive one or more signals from different LEDs in the transmitter array. The TRIP algorithm first estimates the location coordinate of the left shoulder receiver and assigns it a reference position. If the left shoulder receiver is receiving the signal only from one LED, the coordinates of that LED are assigned as a reference position of the left shoulder of the person. But, if the left shoulder receiver receives multiple signals, which means that it is lying in the overlapping light region of two or more LEDs, it finds the centroid of the received coordinates and assigns this centroid as the reference position for the left shoulder. The assignment of reference position for the right shoulder receiver follows the same process. After determining the individual reference positions for both the shoulders, the algorithm calculates the midpoint of the two reference positions and assigns it as the reference position for the persons current position. This is represented in the flow chart for TRIP algorithm in Fig. 2, and for the purpose of differentiation from OBRIP algorithm the flow chart for OBRIP algorithm is also presented in Fig. 1. As stated earlier, the TRIP algorithm helps in overturning the overlapping light beam problem into a plausible accuracy booster.

To perform performance analysis of TRIP algorithm it is simulated in MATLAB where the conditions of a room, with pre-positioned LEDs and a person with optical receivers placed on each of his shoulders, is replicated. The performance analysis has been done considering the distance between both the shoulders (or receivers) as $0.5 \mathrm{~m}$, which is the average shoulder length for an adult human being. To check the accuracy in position estimation the actual position of the person, using the exact positional coordinates of the left and right shoulders, is calculated, and compared to the estimated position to find the error. A Monte-Carlo approach is taken where various random positions of the person are simulated, keeping the distance between the shoulders constant, inside a room with an indoor positioning system and correspondingly the average error in position estimation is calculated. This serves as a very precise scale of measurement for the performance of the TRIP algorithm. Further analysis is done by using a range of values for parameters like light beam radius and number of LEDs in an array and analyzing the output. Beam radius is the maximum distance up to which a prepositioned LED can emit its light with adequate intensity. Beam radius vs. Average error plot for varying number of LEDs in the LED array, Beam radius vs. Average error plot for different room shapes and for different grid size have been drawn, and using these plots the optimal values of important parameters have been determined.

\section{Simulation Setup AND Results}

In the top view snapshot of a room with a OWC-based IPS as shown in Fig. 3, the nine large circles denote the beam area covered by each LED of the prepositioned LED array. The small circles and stars denote some possible positions of the right and left shoulder of a person respectively. Though 25000 such positions have been simulated for the purpose of analysis, but in Fig. 3 only a small number is depicted for purpose of understanding the concept. The room area for simulation is assumed to be $100 \mathrm{~m}^{2}(10 \mathrm{~m} \mathrm{x} 10 \mathrm{~m})$ with a prepositioned LED array with nine LEDs. As can be seen in the figure, the radius of the light beam is greater than the separation between the LEDs so the beam area tends to overlap for adjacent LEDs. As this algorithm uses predetermined reference points to assign a location to an object (or person), a crucial step for the success of this algorithm is to obtain these predetermined reference points. The first set of these points are obtained from the midpoints of the light beam area of the LED which is actually the position coordinates of the LEDs. As we stated earlier, this algorithm uses overlapping light beam regions to its advantage, so the other set of the predetermined reference points are derived by calculating the centroid coordinates of the LEDs with overlapping light beam regions. The other key aspect of this algorithm is the usage of two receivers on a single object (or person) as this is what helps in reducing the error in position estimation. For each of these two receivers, which are separated by a fixed distance, reference position would be calculated separately and their mid-point will give the reference position of the object. The MATLAB simulation proceeds with 25000 uniformly distributed random points generated within a room representing 25000 possible positions of a device inside a room. These illustrate one of the the receivers on the left shoulder of the person. Similarly, for 


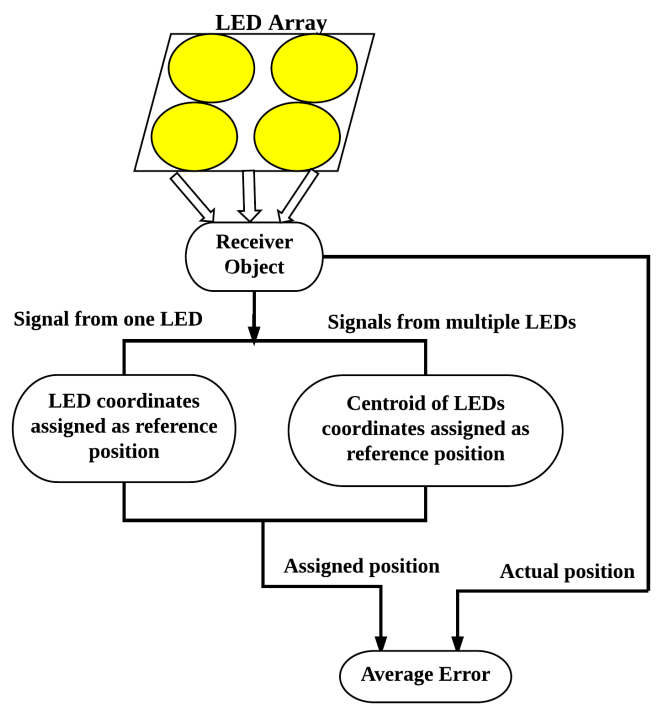

Fig. 1: System flowchart for OBRIP algorithm

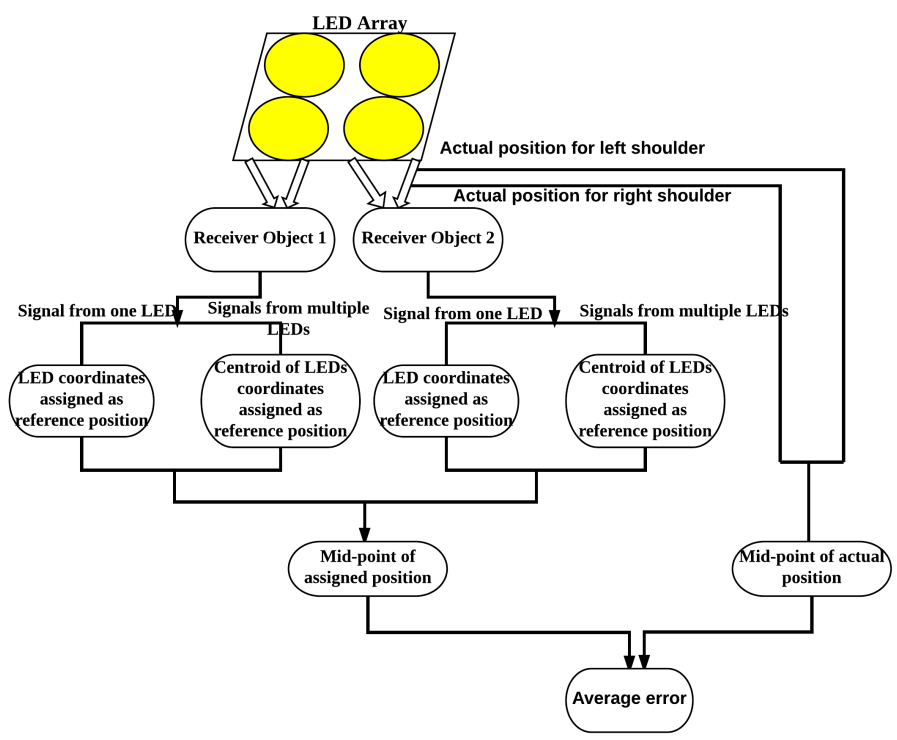

Fig. 2: System flowchart for TRIP algorithm

the right shoulder a new set of 25000 uniformly distributed random points, such that each point is at a fixed distance of $0.5 \mathrm{~m}$ from a unique left shoulder point, is generated. These pairs of random points representing left and right shoulder of a person emulate all the possible scenarios of the position of the person in the room: 1) both shoulders in the same light beam area, 2) both shoulders in different light beam areas, 3) both shoulders not in any light beam area and 4) one shoulder in a light beam area and other not in any light beam area.

Now for each receiver, when it will receive one or more light signals, position estimation would be performed separately. One scenario could be when a receiver receives signal from a single LED, then the LED's position will be assigned as its own reference position. The second scenario could be when it receives signals from multiple LEDs, then the centroid of

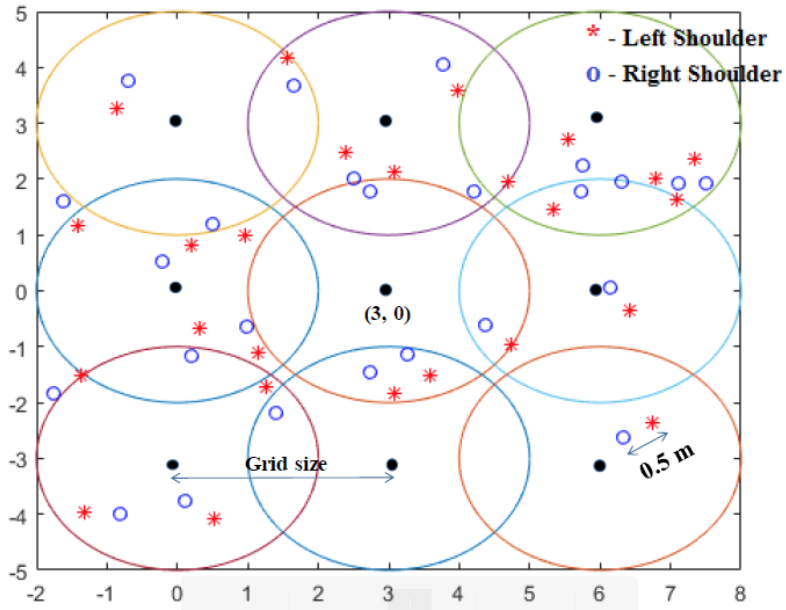

Fig. 3: Top-view of room using OWC-based IPS with two receivers (larger circle represents LED beam area)

the coordinates of all the LEDs it is getting a signal from is assigned as its reference position. In the third scenario, when the receiver receives no light signal from any LED it is assigned a reference position as the coordinates of the center of the room. After estimation for reference position is done for each receiver, the midpoint for the pair of left and right shoulder receivers is calculated and assigned as the final reference position for the person.

To find the optimized values for important parameters like beam radius, number of LEDs and separation between LEDs, the same experiment is repeated by varying the values for these parameters and analyzing the performance of the system. This experiment is also repeated for various room shapes in order to inspect if there is any variation in performance of the system. Equations (1) and (2) are used to find the average error between the actual position and the reference position.

$$
\begin{gathered}
d=\sqrt{\left(x_{1}-x_{2}\right)^{2}+\left(y_{1}-y_{2}\right)^{2}} \\
\bar{E}=\frac{1}{N}\left(\sum_{i=1}^{N} d_{i}\right)
\end{gathered}
$$

- $d_{i}$ is the average distance between the actual position and assigned position

- $\left(x_{1}, y_{1}\right)$ is the assigned reference position

- $\left(x_{2}, y_{2}\right)$ is the actual position (mid point of the random points)

- $\bar{E}$ is the average error

- $N$ is the total number of random points generated

- In this research, $N=25000$

\section{A. Variation of number of LEDs}

The number of prepositioned LEDs in the LED array can play an important role in determining the accuracy of the LEDbased indoor positioning system. For the performance analysis of OBRIP algorithm [19], the number of LEDs in the array is varied from 5 to 9 , and for each combination of number of LEDs the beam radius is also varied from $0.25 \mathrm{~m}$ to $8 \mathrm{~m}$ in 
TABLE I. Average error and optimal beam radius for different number of LEDs (TRIP and OBRIP)

\begin{tabular}{|c|c|c|c|c|}
\hline $\begin{array}{c}\text { No. of } \\
\text { LEDS }\end{array}$ & $\begin{array}{c}\text { Optimal } \\
\text { Radius } \\
\text { (TRIP) }\end{array}$ & $\begin{array}{c}\text { Average } \\
\text { Error } \\
\text { (TRIP) }\end{array}$ & $\begin{array}{c}\text { Optimal } \\
\text { Radius } \\
\text { (OBRIP) }\end{array}$ & $\begin{array}{c}\text { Average } \\
\text { Error } \\
\text { (OBRIP) }\end{array}$ \\
\hline 9 & 2.5 & 0.69 & 2.5 & 1.00 \\
\hline 8 & 2.5 & 0.92 & 2.75 & 1.19 \\
\hline 7 & 2.5 & 1.10 & 2.75 & 1.35 \\
\hline 6 & 2.5 & 1.48 & 2.75 & 1.78 \\
\hline 5 & 2.5 & 1.72 & 2.75 & 2.04 \\
\hline
\end{tabular}

steps of $0.25 \mathrm{~m}$. This helps in finding out the best count of LEDs in the array along with the optimal beam radius value for each combination of number of LEDs. In this research, a similar analysis is conducted for the TRIP algorithm to compare the results with the OBRIP algorithm. Initially, 9 LEDs are placed such that adjacent LEDs are equidistant from each other. Then to decrease the LED count from 9 to 5, one LED is removed at a time such that its distance from the adjacent vertical and horizontal LEDs is $3 \mathrm{~m}$ and distance from diagonally adjacent LEDs is $3 \sqrt{2} \mathrm{~m}$. The process of signal detection is the same as stated in the simulation setup and also shown in Fig. 2. The results of this experiment are exhibited in Fig. 4 and Table I, and from the initial comparison between OBRIP and TRIP algorithm based on these results it can be observed that for both algorithms when the number of LEDs is increased the average error in position estimation decreases, but the overall average error values for each LED combination is smaller in the TRIP algorithm than the OBRIP algorithm. The best number of LEDs for the current system is concluded to be 9 at a beam radius of $2.5 \mathrm{~m}$ as minimum average error of $0.69 \mathrm{~m}$ is observed at this LED count. Increasing the number of LEDs beyond 9 has a small impact on the average error value as shown in Fig. 5, but it would certainly increase the cost of the entire system.

Also, from Fig. 5, the effect of beam radius on the average error can also be visualized for both OBRIP and TRIP algorithm when number of LEDs is 9. It is observed that average error decreases rapidly with increase in the beam radius up to the optimal value of $2.5 \mathrm{~m}$ for TRIP algorithm and further increase in the beam radius results in gradual increase of average error with increasing beam radius. Thus, at a beam radius of 2.5 $\mathrm{m}$ the average error has minimum value. This result is in agreement with the observation from simulation conducted in previous research for OBRIP algorithm [19]. Using the above comparison analysis, it can be safely concluded that for each combination of number of LEDs and beam radius, the TRIP algorithm outperforms OBRIP algorithm giving 30\% less average error values.

\section{B. Variation of room shape}

The next performance evaluation is done on the basis of shape of the room in which the system is installed which might play an important role in determining the accuracy of the positioning algorithm. To test this hypothesis the following room shapes: square $(10 \mathrm{~m} \times 10 \mathrm{~m})$, rectangle $(11.1 \mathrm{~m} \mathrm{x}$ $9 \mathrm{~m})$, circle (radius $=5.64 \mathrm{~m})$, regular hexagon $($ radius $=$

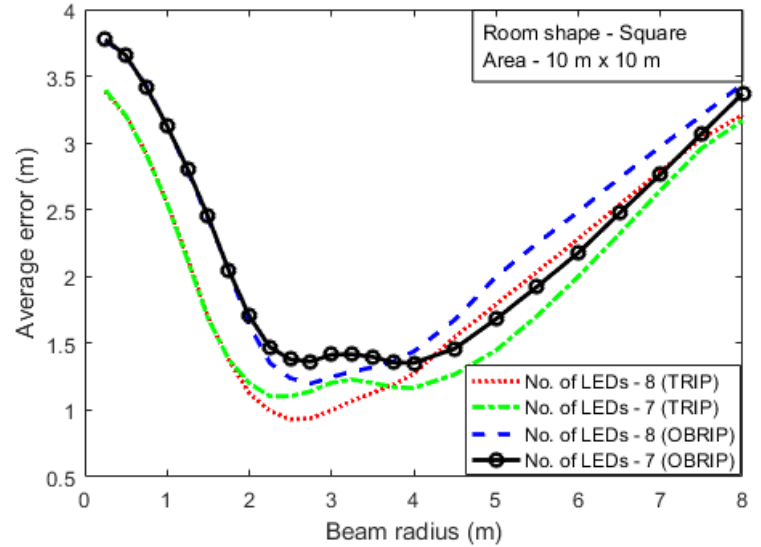

Fig. 4: Average error vs. optimal beam radius for different number of LEDs (TRIP and OBRIP)

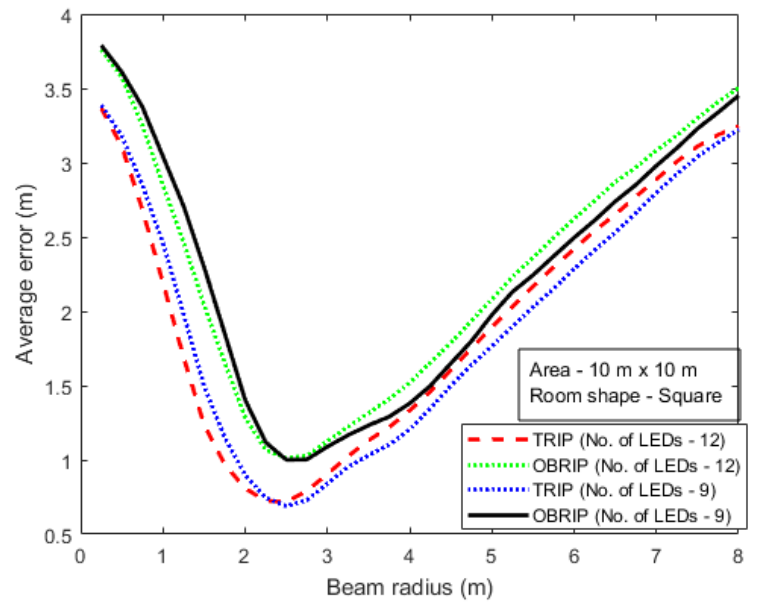

Fig. 5: Average error vs. beam radius for TRIP and OBRIP

$6.21 \mathrm{~m}$ ) and regular pentagon (radius $=7.6 \mathrm{~m}$ ) with constant room area $\left(100 \mathrm{~m}^{2}\right)$ are subjected to the experiment. For each room shape, the number of LEDs in the array is kept nine, and the LEDs are positioned such that the position of the middle LED of the array coincides with the geometric center of the roof of the room. Then, for each shape the beam radius is varied from 0.25 to $8 \mathrm{~m}$ at intervals of 0.25 $\mathrm{m}$ and the average error in position estimation is calculated. The results from the simulation are shown in two separate visualizations in Fig. 6 and Table II. The results show the significance of the room shape in determining the accuracy of the algorithm, where square, hexagon, circle and rectangle shapes give small average error values, but the average error value for the pentagon room shape is much higher. This result is consistent across both OBRIP and TRIP algorithms, but TRIP algorithm gives smaller average error values for each of the simulated shapes by $26 \%-30 \%$.

\section{Variation of the LEDs grid size}

The OWC-based indoor positioning system consists of an array of prepositioned LEDs in which adjacent LEDs have a fixed separation distance between them. The final phase of performance analysis for this research is based of varying 


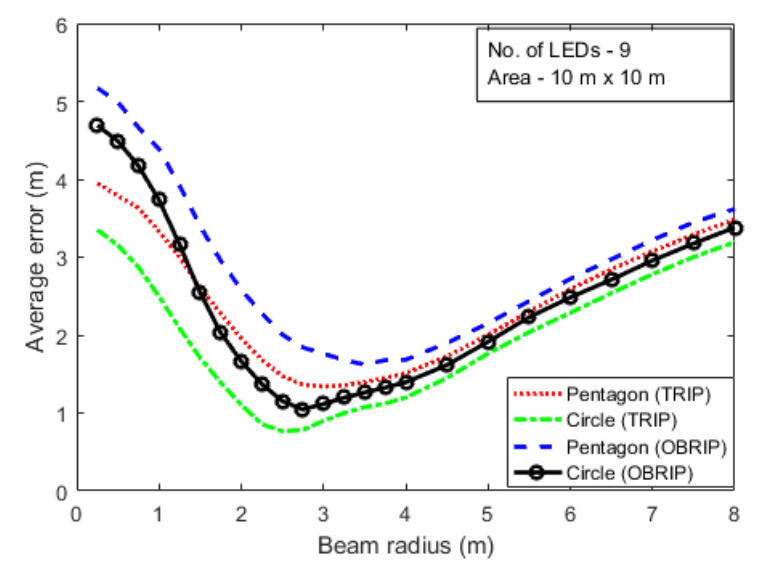

Fig. 6: Average error vs. optimal beam radius for different room shapes (TRIP and OBRIP)

TABLE II. Average error and optimal beam radius for various room shapes (TRIP and OBRIP)

\begin{tabular}{|c|c|c|c|c|}
\hline $\begin{array}{c}\text { Room } \\
\text { shape }\end{array}$ & $\begin{array}{c}\text { Optimal } \\
\text { Radius } \\
\text { (TRIP) }\end{array}$ & $\begin{array}{c}\text { Average } \\
\text { Error } \\
\text { (TRIP) }\end{array}$ & $\begin{array}{c}\text { Optimal } \\
\text { Radius } \\
\text { (OBRIP) }\end{array}$ & $\begin{array}{c}\text { Average } \\
\text { Error } \\
\text { (OBRIP) }\end{array}$ \\
\hline Square & 2.5 & 0.69 & 2.75 & 1.00 \\
\hline Hexagon & 2.75 & 0.76 & 2.75 & 1.02 \\
\hline Circle & 2.5 & 0.76 & 2.75 & 1.05 \\
\hline Rectangle & 2.75 & 0.90 & 2.75 & 1.23 \\
\hline Pentagon & 3 & 1.34 & 3.5 & 1.66 \\
\hline
\end{tabular}

this distance and analyzing the performance of the algorithm and comparing it to the OBRIP algorithm. For the purpose of performance analysis, the separation between adjacent LEDs of the array is varied from $1.5 \mathrm{~m}$ to $5.5 \mathrm{~m}$ in steps of $0.25 \mathrm{~m}$ for all the room shapes mentioned in the previous section with the position of the center LED fixed at the geometrical center of the roof of the room $(3,0)$ as shown in Fig. 3. The remaining experimental setup is same as stated in the simulation setup section. The change in average error is then observed for each room shape on variation of the LED separation distance. From the simulation results for each shape as shown in Fig. 7, Fig. 8 and Fig. 9, it can be observed that for each room shape for a grid size up to $3.5 \mathrm{~m}$ there is a steep decrease in average error with increasing beam radius. Also, from the different lines in these figures which portray different values of grid size, it can be observed that the minimum value of average error obtained at the optimal beam radius point keeps on decreasing with increase in grid size up to a certain value of grid size. Increase in grid size beyond $3.5 \mathrm{~m}$ reduces the rate of change of average error with beam radius, but the minimum value of average error at the optimal beam radius for a grid size is negligible. As before, this result is as obtained from the performance analysis of OBRIP algorithm, but again the TRIP algorithm with smaller average error values is more precise.

\section{CONCLUSION}

OWC technology for indoor positioning systems suffers from a major setback caused by overlapping light beam regions of the transmitting LEDs which leads to decrease in accuracy of the estimated position. The TRIP algorithm proposed in this

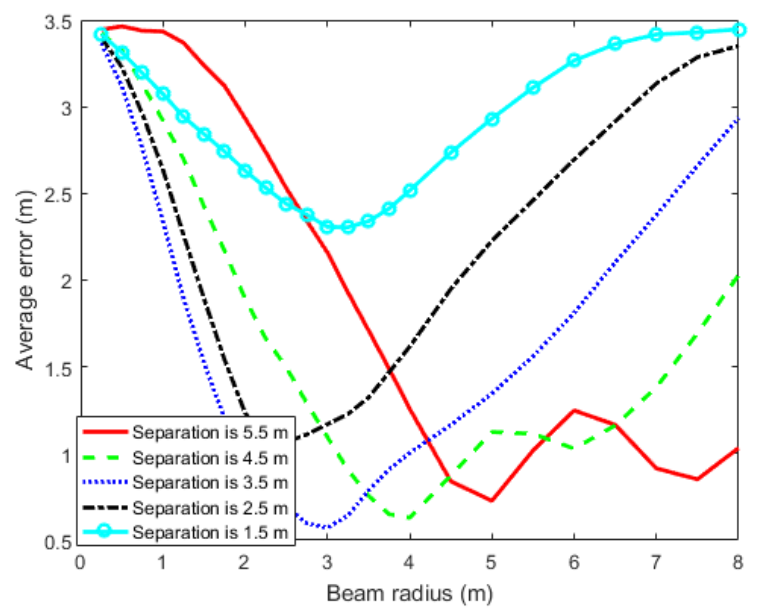

Fig. 7: Average error vs. beam radius for different grid size using TRIP algorithm (Square)

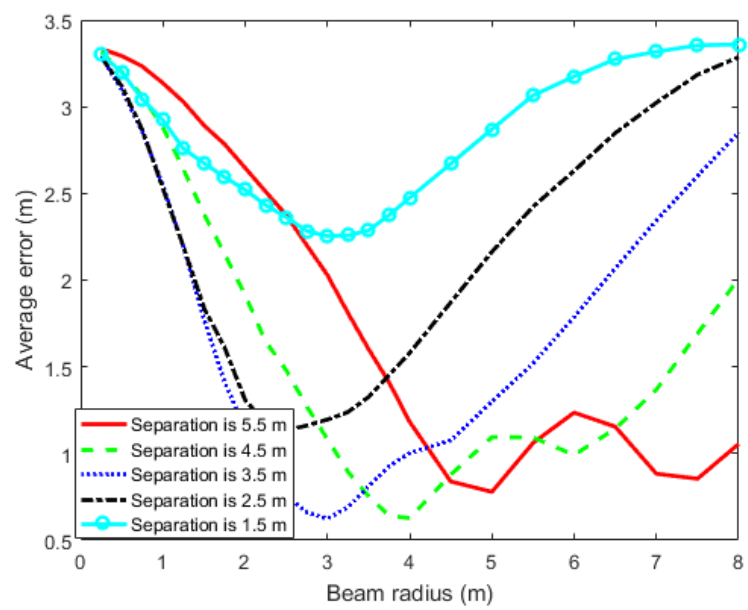

Fig. 8: Average error vs. beam radius in position estimation for different grid size for TRIP algorithm (Hexagon)

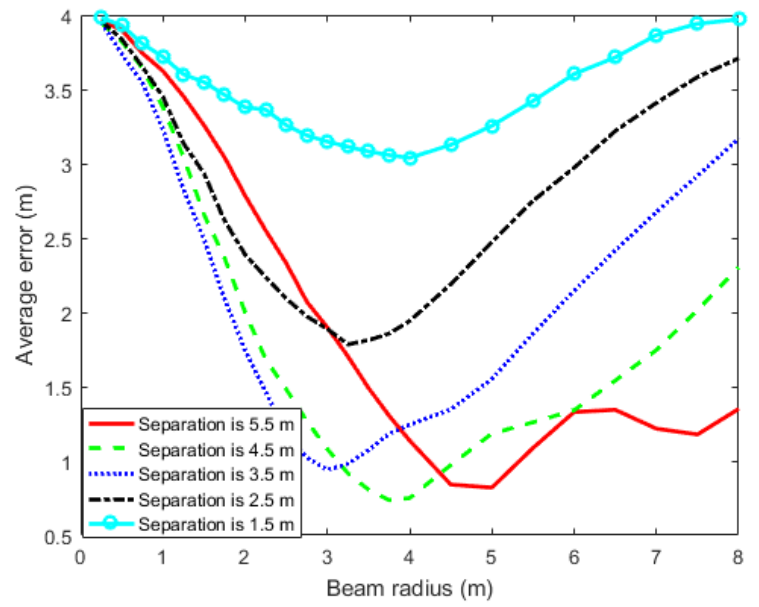

Fig. 9: Average error vs. beam radius for different grid size using TRIP algorithm (Pentagon) 
research advantageously uses this overlapping problem and translates it to increase the overall accuracy of the positioning system. In related research, the OBRIP algorithm is proposed which uses a similar technique for position estimation. But, in TRIP algorithm two receivers are associated with one object which shows tremendous potential to outperform the accuracy of the OBRIP algorithm.

From the performance analysis of TRIP algorithm conducted in this research, the least value of average error in position estimation is observed for a square-shaped room with an array of 9 prepositioned transmitter LEDs having a beam radius of $2.5 \mathrm{~m}$ each, and distance between adjacent LEDs, or grid size being $3.5 \mathrm{~m}$. A further reduction in error is possible when the number of LEDs in the array or number of receivers on the object are increased, but the cost factor overshadows the minuscule improvement in accuracy. Also, from the performance comparison of TRIP and OBRIP algorithm done in this research it can be concluded that the average error in position estimation is $30 \%$ less for TRIP.

Various indoor positioning parameters like beam radius of the LED, number of LEDs in the transmitter array and LED grid size have been varied and performance of the algorithm, in terms of average error in position estimation, has been analyzed for various room shapes. From the results, it is found that radius and average error follow a U-shape relationship which is also depicted in Fig. 5, whereas number of LEDs in an array and average error are inversely related as shown in Table I.

The practical implementation of this algorithm for indoor positioning systems would be a low-cost alternative to existing technologies currently available in the market. Though two receivers are used for implementation of this algorithm, the cost of receivers is relatively low and there is no substantial change in the overall cost of the system when compared to the OBRIP algorithm. TRIP can be used to design and develop a device to track the position of the elderly dementia affected people, or other patients, in a medical facility. It can also be used to track the location of large medical equipment, or members of the medical staff. A possible future research opportunity lies in analyzing the performance of both algorithms in the presence of a line-of-sight propagation channel and confirm their feasibility of their usage under a practical indoor conditions.

\section{ACKNOWLEDGMENT}

The authors would like to express their gratitude towards the Glasgow Caledonian University for making this research possible through their funding, and providing excellent resources and equipment which helped in making the research easier.

\section{REFERENCES}

[1] H. Liu, H. Darabi, P. Banerjee, and J. Liu, "Survey of wireless indoor positioning techniques and systems," IEEE Transactions on Systems, Man, and Cybernetics, Part C (Applications and Reviews), vol. 37, no. 6, pp. 1067-1080, 2007
[2] A. Alarifi, A. Al-Salman, M. Alsaleh, A. Alnafessah, S. Al-Hadhrami, M. A. Al-Ammar, and H. S. Al-Khalifa, "Ultra wideband indoor positioning technologies: Analysis and recent advances," Sensors, vol. 16, no. 5, p. 707, 2016.

[3] D. Dardari, P. Closas, and P. M. Djurić, "Indoor tracking: Theory, methods, and technologies," IEEE Transactions on Vehicular Technology, vol. 64, no. 4, pp. 1263-1278, 2015.

[4] P. Luo, Z. Ghassemlooy, H. Le Minh, A. Khalighi, X. Zhang, M. Zhang, and $\mathrm{C}$. Yu, "Experimental demonstration of an indoor visible light communication positioning system using dual-tone multi-frequency technique," in 3rd International Workshop in Optical Wireless Communications (IWOW), 2014. IEEE, 2014, pp. 55-59.

[5] S. Yang, E. Jeong, and S. Han, "Indoor positioning based on received optical power difference by angle of arrival," Electronics Letters, vol. 50, no. 1, pp. 49-51, 2014.

[6] Z. Zhou, M. Kavehrad, and P. Deng, "Indoor positioning algorithm using light-emitting diode visible light communications," Optical Engineering, vol. 51, no. 8, pp. 085009-1, 2012.

[7] S.-H. Yang and S.-K. Han, "VLC based indoor positioning using singleTx and rotatable single-Rx," in 12th International Conference on Optical Internet 2014 (COIN), 2014. IEEE, 2014, pp. 1-2.

[8] H. Koyuncu and S. H. Yang, "A survey of indoor positioning and object locating systems," IJCSNS International Journal of Computer Science and Network Security, vol. 10, no. 5, pp. 121-128, 2010.

[9] H.-S. Kim, D.-R. Kim, S.-H. Yang, Y.-H. Son, and S.-K. Han, "An indoor visible light communication positioning system using a RF carrier allocation technique," Journal of Lightwave Technology, vol. 31, no. 1, pp. 134-144, 2013.

[10] S.-H. Yang, E.-M. Jung, and S.-K. Han, "Indoor location estimation based on LED visible light communication using multiple optical receivers," IEEE Communications Letters, vol. 17, no. 9, pp. 1834-1837, 2013.

[11] S. Yamaguchi, V. V. Mai, T. C. Thang, and A. T. Pham, "Design and performance evaluation of VLC indoor positioning system using optical orthogonal codes," in IEEE Fifth International Conference on Communications and Electronics (ICCE), 2014. IEEE, 2014, pp. 5459.

[12] A. Yazici, U. Yayan, and H. Yücel, "An ultrasonic based indoor positioning system," in 2011 International Symposium on Innovations in Intelligent Systems and Applications (INISTA). IEEE, 2011, pp. 585-589.

[13] S. De Lausnay, L. De Strycker, J.-P. Goemaere, N. Stevens, and B. Nauwelaers, "Optical CDMA codes for an indoor localization system using VLC," in 3rd International Workshop in Optical Wireless Communications (IWOW), 2014. IEEE, 2014, pp. 50-54.

[14] T. Huang, X. Gao, Y. Guo, S. Li, Q. Li, C. Li, H. Zhu, and Y. Wang, "Visible light indoor positioning fashioned with a single tilted optical receiver," in 14th International Conference on Optical Communications and Networks (ICOCN), 2015. IEEE, 2015, pp. 1-4.

[15] S.-H. Yang, E.-M. Jeong, D.-R. Kim, H.-S. Kim, Y.-H. Son, and S.K. Han, "Indoor three-dimensional location estimation based on LED visible light communication," Electronics Letters, vol. 49, no. 1, pp. 54-56, 2013.

[16] M. Aminikashani, W. Gu, and M. Kavehrad, "Indoor positioning with OFDM visible light communications," in 13th IEEE Annual Consumer Communications \& Networking Conference (CCNC), 2016. IEEE, 2016, pp. 505-510.

[17] W. Zhang, M. S. Chowdhury, and M. Kavehrad, "Asynchronous indoor positioning system based on visible light communications," Optical Engineering, vol. 53, no. 4, pp. 045 105-045 105, 2014.

[18] O. Popoola, F. Ogunkoya, W. Popoola, R. Ramirez-Iniguez, and S. Sinanović, "Indoor localization based on Multiple LEDs Position Estimation," in 17th IEEE International Workshop on Signal Processing Advances in Wireless Communications (SPAWC). IEEE, 2016, pp. 1-6.

[19] M. Ajmani, S. Sinanović, and T. Boutaleb, "Optimal beam radius for LED-based indoor positioning algorithm," in International Conference for Students on Applied Engineering (ISCAE). IEEE, 2016, pp. 357361. 